\title{
Eckpunkte zur Reform des Datenschutzrechts
}

\section{Konferenz, 17./18. März in Stuttgart}

\section{Ein modernes \\ Datenschutzrecht für das 21. Jahrhundert}

\section{Zusammenfassung}

Jeder Mensch soll selbst bestimmen können, wer was wann über ihn weiß. Doch wie soll dieses Recht auf informationelle Selbstbestimmung im Zeitalter der allgegenwärtigen, oftmals unbemerkten $\mathrm{Da}$ tenverarbeitung gewährleistet werden? Die Konferenz der Datenschutzbeauftragten des Bundes und der Länder hat Eckpunkte formuliert, die Grundlage einer Diskussion über eine Reform des Datenschutzrechts sein sollen.

\section{Konkrete Schutzziele und Grundsätze verankern}

Das Bundesdatenschutzgesetz und die Landesdatenschutzgesetze sollten als allgemeingültige datenschutzrechtliche Grundregelungen einen verbindlichen Mindeststandard festlegen. Sie sollten allgemeine Vorgaben enthalten, die als Grundlage aller datenschutzrechtlichen Regelungen und Maßnahmen für öffentliche und nicht-öffentliche Stellen dienen. Ausgehend von den Schutzzielen sollten sanktionsbewehrte Grundsatznormen formuliert werden, die für alle Formen der Datenverarbeitung gleichermaßen gelten. Dies betrifft etwa den Grundsatz der Zweckbindung, also das Prinzip, dass personenbezogene Daten ausschließlich für den Zweck verwendet werden dürfen, für den sie erhoben worden sind. Neu eingeführt werden sollte zudem ein grundsätzliches Verbot der Profilbildung. Die Vorgaben des allgemeinen Datenschutzrechts können - soweit erforderlich - in Bezug auf bestimmte Anwendungsgebiete weiter konkretisiert werden.

\section{Technikneutralen Ansatz schaffen}

Den aus der technologischen Entwicklung resultierenden Gefährdungen sollte durch technikneutrale Vorgaben begegnet werden, die auf konkrete Systeme und Anwendungsfelder durch Auslegung und Normierung konkretisiert werden können. Anhand festgelegter Schutzziele können so einfache, flexible, und praxistaugliche gesetzliche Bedingungen geschaffen werden, die das Grundrecht auf informationelle Selbstbestimmung und das Grundrecht auf Gewährleistung der Vertraulichkeit und Integrität informationstechnischer Systeme durch technischen und organisatorischen Datenschutz sichern.

\section{Betroffenenrechte stärken}

Dreh- und Angelpunkt zur Durchsetzung des Datenschutzes ist der aufmerksame und kritische Betroffene. Die Datenverarbeitung muss für die Betroffenen transparenter werden, etwa indem die Wahrnehmung des Auskunftsanspruchs erleichtert wird. Die Freiwilligkeit der Einwilligung in eine Datenverarbeitung muss gestärkt werden.

\section{Datenschutzrecht internetfähig machen}

Ein modernes Datenschutzrecht muss internetfähig sein. Grundsätzlich muss eine unbeobachtete Kommunikation und Nutzung des Internets gewährleistet werden. Auch sind besondere Schutzmechanismen zur Gewährleistung und Durchsetzung der Datenschutzrechte der Betroffenen im Netz zu schaffen. Nationale Regelungen sollten durch internationale Vereinbarungen flankiert werden.

\section{Mehr Eigenkontrolle statt Zwang}

Datenschutz muss von den verantwortlichen Stellen als eigenes Anliegen begriffen werden. Dies kann etwa durch Einfüh- rung eines freiwilligen Auditverfahrens befördert werden. Daneben müssen die verantwortlichen Stellen dazu verpflichtet werden, durch interne Mechanismen die Einhaltung des Datenschutzes sicherzustellen, etwa durch verbindliche Datenschutzkonzepte.

\section{Stärkung der unabhängigen Datenschutzaufsicht}

Die Unabhängigkeit der Datenschutzaufsicht muss rechtlich, organisatorisch und finanziell abgesichert werden. Eine Fachund Rechtsaufsicht oder die organisatorische Eingliederung in andere Verwaltungseinheiten ist mit der EG-Datenschutzrichtlinie nicht vereinbar. Erforderlich sind auch verstärkte Mitwirkungspflichten der kontrollierten Stellen bei Datenschutzkontrollen.

\section{Wirksamere Sanktionen}

Die immer noch vorhandenen Lücken im datenschutzrechtlichen Sanktionssystem müssen endlich geschlossen werden. Hierfür sollten für die Betroffenen einfach zu handhabende Haftungsansprüche, etwa einen pauschalierten Schadensersatzanspruch, eingeführt werden. Die Zuständigkeiten für die Verfolgung von Ordnungswidrigkeiten sollten bei den jeweiligen Datenschutzbehörden liegen. Auch der Bundesbeauftragte für den Datenschutz und die Informationsfreiheit braucht insoweit wirksame Sanktionsbefugnisse.

\section{Gesetz einfacher und besser lesbar machen}

Das Datenschutzrecht ist durch wiederholte Änderungen und Ergänzungen selbst für Fachleute nur noch schwer verständlich und bedarf auch insoweit der Überarbeitung. Erforderlich sind etwa Änderungen in der Struktur und bei den Definitionen, die zusätzliche Spezialvorschriften entbehrlich machen. 